\title{
Implementation of the new nomenclature in The Astronomical Almanac
}

\author{
Catherine Y. Hohenkerk \\ HM Nautical Almanac Office, UK Hydrographic Office, Admiralty Way, Taunton, Taunton \\ TA1 2DN, UK \\ email: catherine.hohenkerk@ukho.gov.uk
}

\begin{abstract}
This talk charts the implementation of the resolutions of the IAU XXIV General Assembly in 2000 (IAU 2000) in The Astronomical Almanac (AsA).
\end{abstract}

Keywords. reference system, time, standards.

The AsA is a joint publication of the Nautical Almanac Office of the US Naval Observatory (USNO) and the HM Nautical Almanac Office (HMNAO) at the UK Hydrographic Office. All updates must be made in the context of the document into which they are being introduced. The AsA is an annual reference product. It is not a text book but it must maintain standards and be up to date and reliable. It is not at the leading edge, but it must be useful to users and users require continuity. The aspects of the resolutions that affect Section B - Times Scales and Coordinate Systems of the AsA are the implementation of IAU 2000 precession-nutation theory, the recommendation to use what is now called the Celestial Intermediate Origin (CIO) as the origin for right ascension and the Earth rotation angle (ERA). The final version of the IAU2000 precession-nutation software became available in December 2002, and the IAU-SOFA software, the underlying software being used by HMNAO, both equinox and CIO-based, was released in April 2003. For the AsA production this meant implementation in the 2006 edition, with the IAU2000 precession-nutation being used throughout the almanac. It is only when new concepts are fully considered, the software tools developed and an established nomenclature exists, that a new algorithm may successfully be introduced. The areas considered were new tables, new explanations, adding new material into existing pages and finally updating the software, both for the calculations and the new layout.

All the existing quantities are retained including GMST / GAST / GAST-GMST the quantities from the Universal and Sidereal Times pages and the precession and nutation matrix (NPB). The new quantities are the Earth rotation angle (ERA), the equation of the origins (ERA - GAST), the celestial intermediate pole (CIP) and location of the CIO $(X, Y, s)$, and the matrix transformation (C) from the GCRS (Geocentric Celestial Reference System) to the celestial intermediate reference system. No matter how easy it may seem to produce tables of numbers, it is essential to show how the new and existing quantities are used. The explanation of the calculation of intermediate places (CIO-based) and the formation of hour angles has been introduced alongside the equinox-based algorithm for calculating apparent places, which has been extended to calculate hour angles. Although, these processes have different routes, they are described in parallel, and the end results are the same.

The AsA for 2006 was our first almanac to implement the IAU 2000 resolutions, while the 2007 edition is now published and the 2008 edition has just gone to press. USNO Circular 179 by G. Kaplan, at http://aa.usno.navy.mil/publications/docs/Circular 179.html, should be consulted for more detailed explanatory material. 